\title{
Limiting extensibility constitutive model with distributed fibre orientations and ageing of abdominal aorta
}

\author{
Lukáš Horný1*, Marek Netušil ${ }^{2}$, Matěj Daniel ${ }^{1}$ \\ ${ }^{1}$ Czech Technical University in Prague, Faculty of Mechanical Engineering, Technická 4, 166 07, \\ Prague, Czech Republic \\ ${ }^{2}$ Charles University in Prague, Faculty of Mathematics and Physics, Sokolovská 83, 186 75, Prague, \\ Czech Republic \\ *Corresponding author: lukas.horny@fs.cvut.cz, tel. +420 224352690
}

\begin{abstract}
The abdominal aorta is susceptible to age-related pathological changes (arteriosclerosis, atherosclerosis, aneurysm, and tortuosity). Computational biomechanics and mechanobiology provide models capable of predicting mutual interactions between a changing mechanical environment and patho-physiological processes in ageing. However, a key factor is a constitutive equation which should reflect the internal tissue architecture. Our study investigates three microstructurally-motivated invariant-based hyperelastic anisotropic models suitable for description of the passive mechanical behaviour of the human abdominal aorta at a multiaxial state of stress known from recent literature. The three adopted models have also been supplemented with a newly proposed constitutive model (limiting extensibility with fibre dispersion). All models additively decouple the mechanical response of the isotropic (elastin and smooth muscle cells represented by the neo-Hookean term) and the anisotropic (collagen) parts. Two models use exponential functions to capture large strain stiffening ascribed to the engagement of collagen fibres into the load-bearing process. The other two models are based on the concept of limiting extensibility. Perfect alignment of reinforcing fibres with two preferred directions as well as fibre dispersion are considered. Constitutive models are calibrated to the inflation-extension response adopted from the literature based on the computational model of the residually-stressed thick-walled tube. A correlation analysis of determined material parameters was performed to reveal dependence on the age. The results of the nonlinear regression suggest that limiting fibre extensibility is the concept which is suitable to be used for the constitutive description of the aorta at multiaxial stress states and is highly sensitive to ageing-induced changes in mechanical response.
\end{abstract}

Keywords: Abdominal aorta; ageing; anisotropy; constitutive modelling; limiting extensibility; strain energy density. 


\section{INTRODUCTION}

The abdominal aorta is highly susceptible to age-related pathological changes. The most frequent are: arteriosclerosis (calcification, thinning and disruption of elastin membranes in the middle layer of the wall which is accompanied with diameter enlargement and significant stiffening due to a transfer of load-carrying capacity to collagen; Greenwald, 2007; O'Rourke and Hashimoto, 2007; McEniery et al., 2007; Persy and D'Haese, 2009), atherosclerosis (inflammatory plaque formation via lipid accumulation with subsequent calcification on the inner layer; Persy and D'Haese, 2009; Pyle and Young, 2010; Corti and Fuster, 2003), aneurysm (balloon-like expansion with a risk of a rupture; Humphrey and Holzapfel, 2012) and tortuosity (loss of the straightness of the vessel axis; Dougherty and Varro, 2000; Lee et al., 2011; Wenn and Newman, 1990).

Their development seems to be correlated (among other factors) with a mechanical environment specific to the infrarenal part of the aorta where blood flow as well as distribution of intramural stresses are affected by aorto-iliac bifurcation with its complex geometry and mutual non-planarity between the aorta and iliac arteries (Fung, 1997; Thubrikar, 2007; Bäck et al., 2013). At present, computational methods are capable of elucidating the mutual interactions between a changed mechanical environment and an onset or progress of pathological changes (Holzapfel and Ogden, 2010; Wagenseil, 2011; Valentín et al., 2011; Bäck et al., 2013). The key factor limiting the usefulness and reliability of computational models is the constitutive description.

The gradual expansion of the histo-morphometrical and mechanobiological knowledge of the arterial wall has led in recent years to the development of microstructurally-motivated constitutive models. Direct implementation of microstructural information is usually based on the modelling of fibrillar networks of mechanically important constituents (e.g. collagen, elastin, smooth muscle cells). This approach was used by Y. Lanir in his seminal work (Lanir, 1983) and combines the ideas of the density function describing spatial distribution of the orientation of fibrils and the density distribution of their crimping (originally proposed by Decraemer et al., 1980).

Wuyts et al. (1995) used the idea of a statistical description of collagen fibre waviness when interpreting pressure-radius data from 45 thoracic and 20 abdominal human aortas collected by Langewouters et al. (1984). They found a positive correlation between age and Young modulus of collagen fibres in both thoracic and abdominal aortas with a significantly closer dependence in the thoracic part whereas the rate of engagement of collagen fibrils into the load bearing process exhibited a higher correlation with age in the abdominal aorta. Zulliger and Stergiopulos (2007) reanalysed the data of Langewouters et al. (1984) with a 3D constituent-based model and concluded that the ageing of the aorta is neither reflected in a change of elastic constants of elastin and collagen as such nor in the collagen waviness in their model. Nevertheless, the fact that with increased age the strain at which collagen fibres are significantly engaged into the deformation is decreased remains true.

Another approach, perhaps the predominant one, is based on the interpretation of material anisotropy by means of the microstructure employing deformation invariants. G.A. Holzapfel, T.C. Gasser and R.W. Ogden proposed a hyperelastic constitutive model for passive mechanical response of arterial tissue which additively decouples isotropic (elastin, smooth muscle cells) and anisotropic (ascribed to presence of oriented bundles of collagen fibrils) response (Holzapfel et al., 2000). Using additional strain invariants generated by preferred directions in a continuum, they arrived at a form preserving exponential function (to reflect large strain stiffening interpreted as sequential engagement of the collagen into the deformation) originally introduced to biomechanics by Y.C. Fung and confirmed by decades of research to be very successful (Fung, 1967; Fung et al., 1979; Fung and Chuong, 1983; Humphrey, 1995).

Gasser et al. (2006) have recently proposed another invariant-based model which modifies the expression of Holzapfel et al. (2000) by including isotropic contribution into the argument of exponential function. Direct mathematical decoupling between the anisotropic and isotropic contribution enables viewing a large strain stiffening composed of contribution given by a stretching along the preferred direction additionally supported by stretching of fibres dispersed along the 
preferred direction. Recent histo-morphometrical analyses have verified that this is much closer to reality than perfect alignment (Schriefl et al., 2012; Polzer et al., 2013; Horny et al., 2010, 2009).

Adopting the strain energy density function with fibre dispersion, Haskett et al. (2010) analysed the effect of age and location on the biaxial response of the aorta and found age-related stiffening to be the most significant in the abdominal segment. Unfortunately, they did not provide a quantitative correlation with age. Weisbecker et al. (2012) used this model for the descending aorta in a study of cyclic stress-softening. Although they investigated correlations with age, their data sample of abdominal aorta was rather small. The same also applies for in vivo study of Wittek et al. (2013).

In the present study, the above mentioned microstructurally-motivated invariant-based constitutive models (Holzapfel et al., 2000; Gasser et al., 2006) and limiting fibre extensibility model proposed by Horgan and Saccomandi (2005) are calibrated to the inflation-extension behaviour of the human abdominal aorta based on results recently presented by Labrosse et al. (2013). Additionally, a new constitutive model combining the idea of limiting extensibility and fibre dispersion is proposed. Obtained estimates of constitutive parameters are correlated with age and regression equations describing their evolution in ageing are presented.

\section{THEORETICAL FRAMEWORK}

The abdominal aorta will be modelled as an incompressible, hyperelastic, anisotropic residuallystressed homogenous thick-walled tube. Only a passive mechanical response will be considered. Polar cylindrical coordinates will be used in this study and all tensor variables are handled in their physical components.

\subsection{Kinematics of inflation and extension of a tube with residual strains}

During a deformation, let a material particle located originally in point $\Xi=(\rho, \phi, \zeta)$ in the stress-free (reference) configuration be mapped to $\boldsymbol{x}=(r, \theta, z)$ in the deformed configuration. Then, the deformation gradient $\mathbf{F}$ can be obtained as $F_{i K}=\left|\boldsymbol{g}_{i}\right| /\left|\boldsymbol{G}_{K}\right| \cdot \partial x_{i} / \partial \Xi_{K}$ where $\boldsymbol{g}_{i}$ and $\boldsymbol{G}_{K}(i=r, \theta, z$; $K=\rho, \phi, \zeta)$, respectively, are natural covariant base vectors in cylindrical coordinates for the deformed and reference configuration (Humphrey, 2002; Taber, 2004). For incompressible material, $\operatorname{det}(\mathbf{F})=1$ holds. The right Cauchy-Green strain tensor $\mathbf{C}$ and Green-Lagrange strain tensor $\mathbf{E}$ are defined from $\mathbf{F}$ as $\mathbf{C}=\mathbf{F}^{T} \mathbf{F}$ and $\mathbf{E}=1 / 2(\mathbf{C}-\mathbf{I})$, where $\mathbf{I}$ denotes the unit second-order tensor in the material description.

It is well known that tubular segments of arteries excised from a body are not in a stress-free configuration. Residual stresses, ensuring compatibility of deformations of the wall components with different individual stress-free states, take place here (Ambrosi et al., 2011). As the first approximation, it will be assumed that residual stresses are released by radial cut of a cylindrical segment of the aorta (Rachev and Greenwald, 2003; Labrosse et al., 2013; Horny et al., 2013b). Cut radially, a cylindrical segment springs open to a configuration in which its cross-section forms a circular sector which will be considered to be stress-free. The situation is depicted in Figure 1.

Due to the existence of residual strain in the cylindrical segment of the aorta, the kinematics of simultaneous inflation and axial extension described by deformation gradient $\mathbf{F}_{2}$ is considered to be superimposed on closing of an opened up circular sector described by $\mathbf{F}_{1}$. The resulting kinematics is given as $\mathbf{F}=\mathbf{F}_{2} \mathbf{F}_{1}$. Equations (1) and (2), respectively, describe motions from stress-free to closed but unloaded, and from residually stressed to a loaded configuration.

$$
\begin{array}{lll}
R=R(\rho) & \Theta=\frac{2 \pi}{2 \pi-2 \alpha} \phi & Z=\delta \zeta \\
r=r(R) & \theta=\Theta & \mathrm{z}=\lambda Z
\end{array}
$$


According to Figure $1, \rho$ here denotes the variable radius in an undeformed configuration $\left(\rho_{i} \leq \rho \leq \rho_{\mathrm{o}}\right), \alpha$ is the opening angle and $\delta$ is an axial stretch accompanying closing to cylindrical geometry described in coordinates $R, \Theta$, and $Z$. Subsequent pressurisation of the tube (with closed ends) leads to a change of the radius from $R$ to $r\left(r_{i} \leq r \leq r_{o}\right)$ and a change of the length described by axial stretch $\lambda$. Equations (1) and (2) imply that both axial stretches $\delta$ and $\lambda$ are assumed to be uniform along the tube. Deformation gradients related to motions (1) and (2) are in (3a) and (3b), respectively. Final deformation gradient $\mathbf{F}$ is of the form (4).

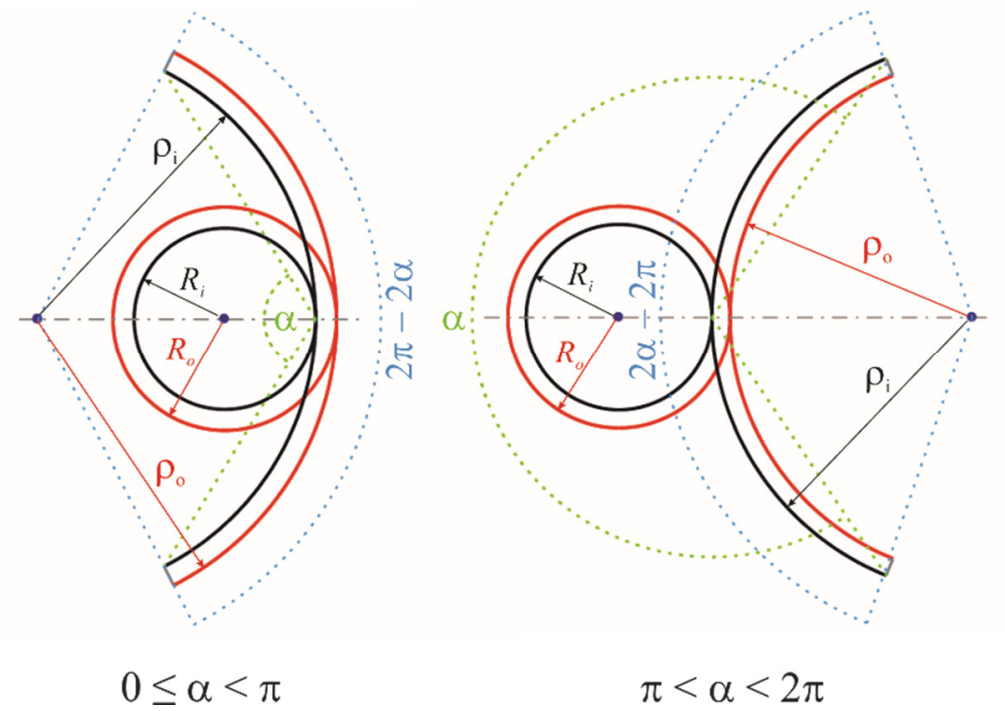

Figure 1. Reference (stress-free) and residually-stressed (unloaded) configurations for opening angle $\alpha \in[0 ; \pi)$ in the left and $\alpha \in(\pi, 2 \pi)$ in the right panel. The arrangement of arterial rings in the figure mimics conditions in an experimental measurement of the opening angle when certain points of the ring have to be fixed. Note that in 3D, an application of residual stress is accompanied with small axial (out-of-plane in the figure) stretch $\delta$.

$$
\begin{aligned}
& \mathbf{F}_{1}=\left(\begin{array}{ccc}
\frac{\partial R(\rho)}{\partial \rho} & 0 & 0 \\
0 & \frac{\pi}{\pi-\alpha} \frac{R(\rho)}{\rho} & 0 \\
0 & 0 & \delta
\end{array}\right) \quad \mathbf{F}_{2}=\left(\begin{array}{ccc}
\frac{\partial r(R)}{\partial R} & 0 & 0 \\
0 & \frac{r(R)}{R} & 0 \\
0 & 0 & \lambda
\end{array}\right) \\
& \mathbf{F}=\mathbf{F}_{2} \mathbf{F}_{1}=\left(\begin{array}{ccc}
\lambda_{r \rho} & 0 & 0 \\
0 & \lambda_{\theta \phi} & 0 \\
0 & 0 & \lambda_{z \zeta}
\end{array}\right)=\left(\begin{array}{ccc}
\frac{\partial r(\rho)}{\partial \rho} & 0 & 0 \\
0 & \frac{\pi}{\pi-\alpha} \frac{r(\rho)}{\rho} & 0 \\
0 & 0 & \lambda \delta
\end{array}\right)
\end{aligned}
$$


Since volume-preserving motion is assumed, $\operatorname{det}\left(\mathbf{F}_{1}\right)=\operatorname{det}\left(\mathbf{F}_{2}\right)=\operatorname{det}(\mathbf{F})=1$. It provides equations decreasing the number of independent components of deformation gradients to two. It will also be useful to express the incompressibility condition by means of inner and outer radii because it will be used within integration in equilibrium equations. The equation (5a) applies for the closing of the circular sector and (5b) for the inflation-extension of the tubular segment. Combining both equations, one obtains volume-preservation condition during pressurisation with respect to a stress-free state.

$$
(\pi-\alpha)\left(\rho_{o}^{2}-\rho_{i}^{2}\right)=\pi\left(R_{o}^{2}-R_{i}^{2}\right) \delta \quad\left(R_{o}^{2}-R_{i}^{2}\right)=\left(r_{o}^{2}-r_{i}^{2}\right) \lambda
$$

At this point, one important note is necessary. Data from Labrosse et al. (2013) will be used here as input parameters. In their experiments, some aortas exhibited opening angle $\alpha \in(\pi, 2 \pi)$; see Table 1 . Figure 1 depicts both $\alpha \in[0 ; \pi)$ and $\alpha \in(\pi, 2 \pi)$ situations separately. It is evident that when the opening geometry is passing through $\alpha=\pi$, the inner and outer radii of the circular sector mutually change their roles (which is a consequence of the changed position of the centre of curvature). Labrosse et al. (2013) pointed out that in such a case, the radii are negative which implies that inequality $\rho_{i} \leq \rho \leq \rho_{o}$ still holds. However, the term $\left(\rho_{o}^{2}-\rho_{i}^{2}\right)$ in the equation (5a) will be negative. The negativity of $\left(\rho_{o}^{2}-\rho_{i}^{2}\right)$ is nevertheless "compensated" by negativity of the term $(\pi-\alpha)$ and obtained volume is a positive real number. A similar situation occurs in $\lambda_{\theta \phi}$. Negativity of $\rho$ is for $\alpha \in(\pi, 2 \pi)$ "compensated" by negativity of $(\pi-\alpha)$ and hence positive principle stretch is obtained.

\subsection{Constitutive models}

The abdominal aorta will be considered as a homogenous incompressible hyperelastic material. The stress-strain relation is thus obtained by differentiating elastic potential (strain energy density per unit reference volume) $W$ with respect to the strain tensor (6). In the equation (6), $\sigma$ is the Cauchy stress tensor and $p$ is an undetermined multiplier induced by the incompressibility constraint.

$\sigma=\mathbf{F} \frac{\partial W}{\partial \mathbf{E}} \mathbf{F}^{T}-p \mathbf{I}$

Within our study, five different models for $W$ will be used. The first of them is the model proposed by Guccione et al. (1991) $W_{G M W}$, eq. (7), recently used in an experimental characterisation of multiaxial mechanical behaviour of the abdominal aorta by Labrosse et al. (2013). The remaining four models will be calibrated to predictions obtained by use of material and geometrical parameters adopted from Labrosse et al. (2013) and equilibrium equations. In (7), $c_{0}$ is stress-like material parameter and $c_{1}$ and $c_{2}$ are dimensionless parameters.

$W_{G M W}=\frac{c_{0}}{2}\left(e^{c_{1} E_{\rho \rho}^{2}+c_{2}\left(E_{\zeta \zeta}^{2}+E_{\rho \rho}^{2}\right)}-1\right)$

The first model, of which material parameters will be estimated, is $W_{H G O}$ proposed by Holzapfel et al. (2000). This model operates with the idea of isotropic matrix (elastin, smooth muscle cells in passive state) described in (8) with the neo-Hookean term and two families of reinforcing fibres (collagen) which generate anisotropic response captured in a continuum by two preferred directions (exponential terms in (8)).

$W_{H G O}=\frac{\mu}{2}\left(I_{1}-3\right)+\sum_{j=4,6}\left(\frac{k_{1}}{2 k_{2}} e^{k_{2}\left(I_{j}-1\right)^{2}}-1\right)$

Here $I_{1}\left(I_{1}=\lambda_{r \rho}^{2}+\lambda_{\theta \phi^{2}}+\lambda_{z} \zeta^{2}\right)$ is the first principal invariant of $\mathbf{C}$ and $\mu[\mathrm{Pa}]$ is the infinitesimal shear modulus of the isotropic matrix. $k_{1}\left(k_{1}>0\right.$, [Pa]) expresses how important the fibre contribution is, and $k_{2}\left(k_{2}>0\right)$ is dimensionless and governs the abruptness of the stiffening. In arguments of exponential functions, $I_{4}$ and $I_{6}$ are strain invariants induced by the existence of preferred directions in a continuum. Let these two directions are aligned with unit reference vectors $\boldsymbol{M}=(0, \cos (\beta), \sin (\beta))$ 
and $N=(0, \cos (-\beta), \sin (-\beta))$. That is to say, they create helices symmetrically disposed with respect to circumferential axis by angle $\pm \beta$ in the surfaces of the constant radius. Invariants $I_{4}$ and $I_{6}$ then can be obtained as $I_{4}=M(\mathbf{C M})$ and $I_{6}=N(\mathbf{C N})$ and equal to the squares of stretches in the preferred directions. The equation (9) holds in our specific case (diagonal $\mathbf{F}$ and independence of radial coordinate).

$$
I_{4}=I_{6}=\lambda_{\theta \phi}^{2} \cos ^{2}(\beta)+\lambda_{z \zeta}^{2} \sin ^{2}(\beta)
$$

It is worth noting that the same parameters $k_{1}$ and $k_{2}$ for the two families of preferred directions and the symmetry in $\pm \beta$ induce a behaviour called local orthotropy (Holzapfel, 2000). Although there are further invariants generated by preferred directions, we will not, in accordance with Holzapfel et al. (2000), include them into the strain energy density models treated in our present study.

Gasser et al. (2006) modified strain energy density (8) to account for the contribution of collagen fibres which are not perfectly aligned with unit vectors $\boldsymbol{M}$ and $N$ replacing $I_{4}$ and $I_{6}$ by $K_{4}$ and $K_{6}(10)$. The interpretation of $\mu, k_{1}$ and $k_{2}$ in (10) remains the same as in (8).

$$
\begin{aligned}
& W_{G O H}=\frac{\mu}{2}\left(I_{1}-3\right)+\sum_{j=4,6}\left(\frac{k_{1}}{2 k_{2}} e^{k_{2}\left(K_{j}-1\right)^{2}}-1\right) \\
& K_{j}=\kappa I_{1}+(1-3 \kappa) I_{j} \quad j=4,6,
\end{aligned}
$$

where $K_{4}$ and $K_{6}$ are generalised invariants, and $\kappa$ is a structural parameter which measures the degree of dispersion $(0 \leq \kappa<1 / 3)$. Considering (11), it is clear that the limits of $\kappa$ correspond to two border situations: $\kappa=0$ implies perfect alignment with preferred directions and isotropy is obtained for $\kappa=1 / 3$. Gasser et al. (2006) showed that one-to-one correspondence between the domain of $\kappa$ (at physically admissible situations) and concentration parameter governing the character of $\pi$-periodic von Mises probability density distribution function does exist (see also the discussion in Holzapfel and Ogden, 2010). This result provides a quantitative characterisation of fibre dispersion.

The third model fitted to the aortic mechanical response in our study will be $W_{H S}$ introduced in Horgan and Saccomandi (2005). It belongs to a different class of models than mentioned above. $W_{G M W}$, $W_{H G O}$ and $W_{G O H}$ employ exponential function which was introduced by Y.C. Fung originally to fulfil differential equation describing the dependence of elastic modulus (derivative of the stress with respect to the strain) on applied stress (in case of uniaxial stress state; Fung, 1967). $W_{H S}$, however, belongs to the class of so-called limiting extensibility models inspired by the kinematics of macromolecular chains. In the simplest case (freely jointed chain), we can imagine a macromolecule as the chain of rigid monomers jointed by frictionless joints where monomers can freely rotate. It is clear that there exist a maximum of end-to-end distance equal to the number of monomers multiplied by their length. This idea is very simple and thus many refinements were suggested. Their details are, however, beyond our scope (for details see articles cited below or the classical textbook by L.R.G. Treloar, 1975). A freely jointed chain is used only to sketch what gives rise to the principle of finite extensibility.

A.N. Gent (1996) suggested a simple invariant-based mathematical form of strain energy density function reflecting the phenomenon of limiting extensibility. It is $W_{G}$ expressed in equation (12). In the context of arterial biomechanics, this model has recently been discussed in Horgan and Saccomandi (2003a), Ogden and Saccomandi (2007), and Horny et al. (2013a) have used it to describe age-related changes in the biomechanics of the abdominal aorta.

$$
W_{G}=-\frac{\mu J_{m}}{2} \ln \left(1-\frac{I_{1}-3}{J_{m}}\right) \wedge \quad I_{1}<J_{m}+3
$$


Here $\mu(\mu>0)$ is the infinitesimal shear modulus, and $J_{m}\left(J_{m}>0\right)$ is a dimensionless limiting extensibility parameter which restricts admissible deformations to the domain given by condition $\lambda_{r \rho}{ }^{2}+\lambda_{\theta \phi}{ }^{2}+\lambda_{z \zeta} \zeta^{2}=I_{1}<J_{m}+3$. When $J_{m} \rightarrow \infty, W_{G}$ is reduced into the neo-Hookean model.

The model (12) is, however, isotropic and thus unsuitable to describe aortic response under multiaxial state of stress. Anisotropic extension of $W_{G}$ to the case of one family of reinforcing fibres (transversely isotropic behaviour) was introduced in Horgan and Sacomandi (2005). Here, in the context of arterial mechanics, we will use the extension to two preferred directions, $W_{H S}$ (13), which may be microstructurally understood in the same way as (8); isotropic matrix and two families of reinforcing collagen fibres. This fact gives $W_{H S}$ its denotation as a limiting fibre extensibility model. Admissible deformations are now restricted by condition $\lambda_{\theta \phi}^{2} \cos ^{2}(\beta)+\lambda_{z} \zeta^{2} \sin ^{2}(\beta)=I_{4}=I_{6}<J_{m}^{1 / 2}+1$. It is again assumed that both families of fibres are mechanically equivalent.

$W_{H S}=\frac{\mu}{2}\left(I_{1}-3\right)-\sum_{j=4,6}\left(\frac{v J_{m}}{2} \ln \left(1-\frac{\left(I_{j}-1\right)^{2}}{J_{m}}\right)\right) \wedge \quad\left(I_{j}<\sqrt{J_{m}}+1 \quad j=4,6\right)$

When limiting extensibility is relaxed, i.e. $J_{m} \rightarrow \infty$, the sum of logarithmic terms in (13) will reduces to $1 / 2 v\left(I_{4}-1\right)^{2}+1 / 2 v\left(I_{6}-1\right)^{2}$. The parameter $v$ [Pa] functions as a measure of anisotropy (it is a weight of the fibres' contribution to the strain energy). Horgan and Saccomandi (2002, 2003b, 2005) also showed that the logarithmic terms of the above mentioned strain energy density functions can be obtained when strain energy is expanded as a function of deformations invariants instead of in polynomials (as was developed by R.S. Rivlin) by rational functions. Further details of limiting extensibility models can be found e.g. in Horgan and Saccomandi (2006), or Horgan and Murphy (2007).

The last material model used in our study, $W_{H N D}$, is based on the introduction of the idea of fibre dispersion into the limiting extensibility model (13). In (14), squares of stretches along the directions of reinforcing fibres are replaced by generalised invariants (11). $\mu, v, J_{m}, \beta$ and $\kappa$, which are the material parameters, have the same meaning as in above mentioned equations and admissible deformations are now restricted by the condition (15).

$$
\begin{aligned}
& W_{H N D}=\frac{\mu}{2}\left(I_{1}-3\right)-\sum_{j=4,6}\left(\frac{v J_{m}}{2} \ln \left(1-\frac{\left(K_{j}-1\right)^{2}}{J_{m}}\right)\right) \wedge\left(K_{j}<\sqrt{J_{m}}+1 \quad j=4,6\right) \\
& K_{j}=\kappa I_{1}+(1-3 \kappa) I_{j} \quad j=4,6
\end{aligned}
$$

\subsection{Equilibrium of thick-walled tube}

Non-trivial equilibrium equations for inflation-extension behaviour of the closed hyperelastic incompressible thick-walled tube with substituted constitutive model are expressed in (16) for radial direction, and in (17) for axial direction. Their form is adopted from Holzapfel and Ogden (2010), Labrosse et al. (2013), Horny et al. (2013b). Detailed derivation can be found in Matsumoto and Hayashi (1996). These equations apply when boundary conditions $\sigma_{r r}\left(r_{i}\right)=-P$ and $\sigma_{r r}\left(r_{o}\right)=0$ are used.

$$
P=\int_{r_{i}}^{r_{o}} \lambda_{\theta \phi} \frac{\partial \hat{W}}{\partial \lambda_{\theta \phi}} \frac{d r}{r}
$$


$F_{\text {red }}=\pi \int_{r_{i}}^{r_{\rho}}\left(2 \lambda_{z \zeta} \frac{\partial \hat{W}}{\partial \lambda_{z \zeta}}-\lambda_{\theta \phi} \frac{\partial \hat{W}}{\partial \lambda_{\theta \phi}}\right) r d r$

$P$ here denotes internal pressure and $F_{\text {red }}$ is axial prestretching force (acting on closed ends of the tube additionally to intraluminal pressure $P$ ). In our specific case, $F_{\text {red }}=0$ because Labrosse et al. (2013) conducted their experiments without prestretching force. In equations (16) and (17), $\hat{W}$ denotes the strain energy density function written in components of $\mathbf{F}$ and with $\lambda_{r \rho}$ substituted by $1 /\left(\lambda_{\theta \phi} \lambda_{z \zeta}\right)$ from incompressibility condition.

Table 1. Input parameters and estimated stress-free geometry. ID of the samples is in the form indicating sex and age [years]. Input parameters are adopted from Labrosse et al. (2013); $R_{i}$ - inner radius in unloaded configuration, $H$ - thickness in unloaded configuration, and $c_{0}, c_{1}$, and $c_{2}$ are material constants in $W_{G M W} . \rho_{i}$ and $\rho_{o}$ denote computed stress-free radii of opened up circular sectors. $\delta$ denotes axial stretch achieved in closing of the opened sector. Note that negativity of $\rho_{i}$ and $\rho_{o}$ is the consequence of the changed position of the centre of curvature (see Figure 1).

\begin{tabular}{|c|c|c|c|c|c|c|c|c|c|c|c|c|c|c|c|c|c|}
\hline ID & F49 & F50 & F63 & F65 & M38 & M42 & M57 & M60 & M61a & M61b & M66 & M67a & M67b & M70a & M70b & M71 & M77 \\
\hline $\begin{array}{l}\text { Opening } \\
\text { angle }\left[{ }^{\circ}\right]\end{array}$ & 252 & 323 & 96 & 248 & 117 & 125 & 322 & 156 & 270 & 335 & 253 & 118 & 174 & 208 & 201 & 118 & 135 \\
\hline $\begin{array}{c}R_{i} \\
{[\mathrm{~mm}]}\end{array}$ & 5.9 & 6.7 & 5.4 & 6.2 & 5.3 & 6.5 & 7.5 & 6.3 & 7.7 & 7.3 & 7.2 & 8 & 7.9 & 7.1 & 7.4 & 10 & 7 \\
\hline $\begin{array}{c}H \\
{[\mathrm{~mm}]}\end{array}$ & 1.51 & 1.14 & 0.96 & 1.21 & 1.22 & 1.56 & 1.28 & 1.69 & 1.22 & 1.62 & 1.78 & 1.58 & 1.26 & 1.23 & 1.64 & 1.72 & 1.5 \\
\hline $\begin{array}{c}c_{0} \\
{[\mathrm{kPa}]}\end{array}$ & 8.4 & 8.4 & 23 & 1.6 & 14.7 & 41.8 & 0.8 & 7.6 & 2.4 & 2.3 & 9.4 & 3.5 & 2.2 & 14 & 1.8 & 17 & 1.2 \\
\hline $\begin{array}{l}c_{1} \\
{[-]}\end{array}$ & 5.09 & 15.21 & 4.07 & 9.26 & 3.04 & 1.54 & 6.74 & 2.96 & 37.53 & 6.82 & 7.81 & 24.47 & 56.69 & 16.09 & 18.62 & 13 & 41.08 \\
\hline $\begin{array}{c}c_{2} \\
{[-]} \\
\end{array}$ & 8.18 & 9.67 & 7.2 & 11.77 & 7.38 & 1.44 & 12.44 & 10.23 & 34.01 & 19.16 & 12.5 & 27.9 & 41.66 & 7.38 & 35.99 & 11.85 & 49.51 \\
\hline $\begin{array}{c}\rho_{i} \\
{[\mathrm{~mm}]}\end{array}$ & -17.34 & -9.72 & 12.10 & -18.59 & 16.20 & 23.00 & -10.95 & 52.16 & -17.22 & -10.22 & -20.77 & 24.68 & 255.2 & -50.23 & -70.93 & 30.63 & 30.17 \\
\hline $\begin{array}{c}\rho_{o} \\
{[\mathrm{~mm}]}\end{array}$ & -15.82 & -8.58 & 13.06 & -17.37 & 17.42 & 24.56 & -9.67 & 53.87 & -16.00 & -8.60 & -18.99 & 26.27 & 256.5 & -49.00 & -69.27 & 32.36 & 31.68 \\
\hline $\begin{array}{c}\delta \\
{[-]}\end{array}$ & 1.0019 & 1.0003 & 1.0006 & 1.0017 & 1.0010 & 1.0039 & 1.0000 & 1.0017 & 1.0010 & 0.9998 & 1.0018 & 1.0022 & 1.0047 & 1.0051 & 1.0033 & 1.0020 & 1.0034 \\
\hline
\end{tabular}

\subsection{Inflation-extension behaviour obtained from $W_{G M W}$}

Labrosse et al. (2013) have recently published the results of 17 inflation-extension tests conducted ex vivo with human abdominal aortas. Their material and geometrical parameters $\left(c_{0}, c_{1}\right.$ and $c_{2}$ for $W_{G M W}$; $R_{i}, H$ and $\alpha$ for geometry) are presented in Table 1 and will be used to generate mechanical response. This response will provide the data which subsequently will be fitted by $W_{H G O}, W_{G O H}, W_{H S}$ and $W_{H N D}$ models.

The simulation is performed in two steps. Since Labrosse et al. (2013) did not quote stress-free dimensions, they must be found in the first step. The procedure involves incompressibility condition and equations (16) and (17) expressed using $\mathbf{F}_{1}$. In the second step, the same equations in deformation described by $\mathbf{F}=\mathbf{F}_{2} \mathbf{F}_{1}$ are used to compute $r_{i}, r_{o}$ and $\lambda_{z \zeta}$.

The problem was formulated as a minimisation of the objective function $Q^{S I M}$ (18) subjected to the constraint $F_{r e d}=0$. In (18), $P$ denotes pressure given by (16). For $i=0$, the first step in which $\rho_{i}, \rho_{o}$, and $\delta$ are solved for, is obtained. $i=1,2, \ldots, 20 \mathrm{kPa}$ corresponds to the pressurisation of the tube. The whole procedure is expressed in (19). Integrals for $P$ and $F_{\text {red }}$ with substituted (7) were handled numerically with 200 integration points per thickness of the tube in Maple 17. NPLSolve (nonlinear programming) command was used. The square in (18) ensures a convexity of the problem. 


$$
\begin{aligned}
& Q^{S I M}(i)=(P-i)^{2} \quad \text { for } i=0,1,2, \ldots, 20 \mathrm{kPa} \\
& \left\{\operatorname{Minimize}\left(Q^{S I M}(i)\right) \wedge F_{\text {red }}(i)=0\right\}_{i=0}^{20} \longrightarrow\left\{r_{i}(i), r_{o}(i), \lambda_{z \zeta}(i)\right\}_{i=0}^{20}
\end{aligned}
$$

\section{5 $W_{H G O}, W_{G O H}, W H S$ and $W_{H N D}$ parameters estimation}

Simulated mechanical response (2.4) was used in the nonlinear regression to obtain estimates of material parameters for $W_{H G O}, W_{G O H}, W_{H S}$ and $W_{H N D}$. Similar to (2.4), it was also based on the minimisation of the constrained objective function, $Q^{R E G}(20)$. Here $P^{E X P}$ and $P^{M O D}$, respectively, denote prescribed inflation pressures and pressures predicted by (16) with yet unknown material parameters and the geometry substituted from (2.4). $F_{\text {red }}{ }^{M O D}$ denotes predicted axial force. $q_{F}$ expresses borders of axial force. Note that, theoretically $F_{\text {red }}$ is zero, which is however satisfied with some numerical accuracy. The problem was again solved in Maple 17 employing the NLPSolve command and 200 integration points per thickness of aortic wall were used. We would like to point out that in contrast to (19), where one objective function subjected to the constraining equation is minimised 21times, (21) is the system of one objective function subjected to 21 double-sided inequalities.

$$
Q^{R E G}=\sum_{i=0}^{n=20}\left(P_{i}^{E X P}-P_{i}^{M O D}\right)^{2}
$$

$\operatorname{Minimize}\left(Q^{R E G}\right) \wedge\left\{-q_{F}<F_{\text {red }}^{M O D}(i)<q_{F}\right\}_{i=0}^{20} \quad \longrightarrow \quad\{$ material parameters $\}$

\subsection{Correlation with age and goodness of fit}

For each aorta, goodness of fit obtained in nonlinear regression was evaluated by means of the coefficient of determination $R_{C D}{ }^{2}$ computed for predicted pressures; $R_{C D}^{2}=1-$ (residual sum of squares)/(total sum of squares). The linear correlation coefficient $R_{L C}$ was computed for all sets of identified parameters to assess their dependence on age. It was supplemented by the test of the hypothesis $H_{0}: R_{L C}=0$ (against alternative $H_{A}: R_{L C} \neq 0$ ) based on the statistics $T=R_{L C}\left[(17-2) /\left(1-R_{L C}^{2}\right)\right]^{1 / 2}$. Shapiro-Wilk test was employed to test the hypothesis that the data follows normal distribution. Results were considered to be statistically significant at the level 0.05 . Some elementary functions $\left(a \cdot x+b, a \cdot \exp (b \cdot x), a \cdot \ln (x)+b, a \cdot x^{b}, a^{b x}\right)$ were used to describe dependence of estimated material parameters on age $(x=$ age [years]). The capability to predict agerelated changes in material parameters was assessed with $R_{C D}{ }^{2}$.

\section{RESULTS}

\subsection{Simulated response}

The results of the 17 analytical inflation-extension simulations of human abdominal aorta were computed and subsequently used to be fitted by other four models of the strain energy density function. Unloaded dimensions and material parameters adopted from the literature (Labrosse et al., 2013) are listed in Table 1. Computed stress-free radii and axial stretch accompanying motion from stress-free to unloaded geometry are also listed here. Obtained values for radii and axial stretches were subsequently substituted to the equations (16) and (17) to check the accuracy of the solution. It was found that in all cases (17 aortas and 21 internal pressures), the difference between prescribed 
pressure and simulated response satisfied lprescribed - simulated $<0.0000001 \mathrm{kPa}$ and maximum difference of computed reduced axial force from zero was $0.0002 \mathrm{mN}$.
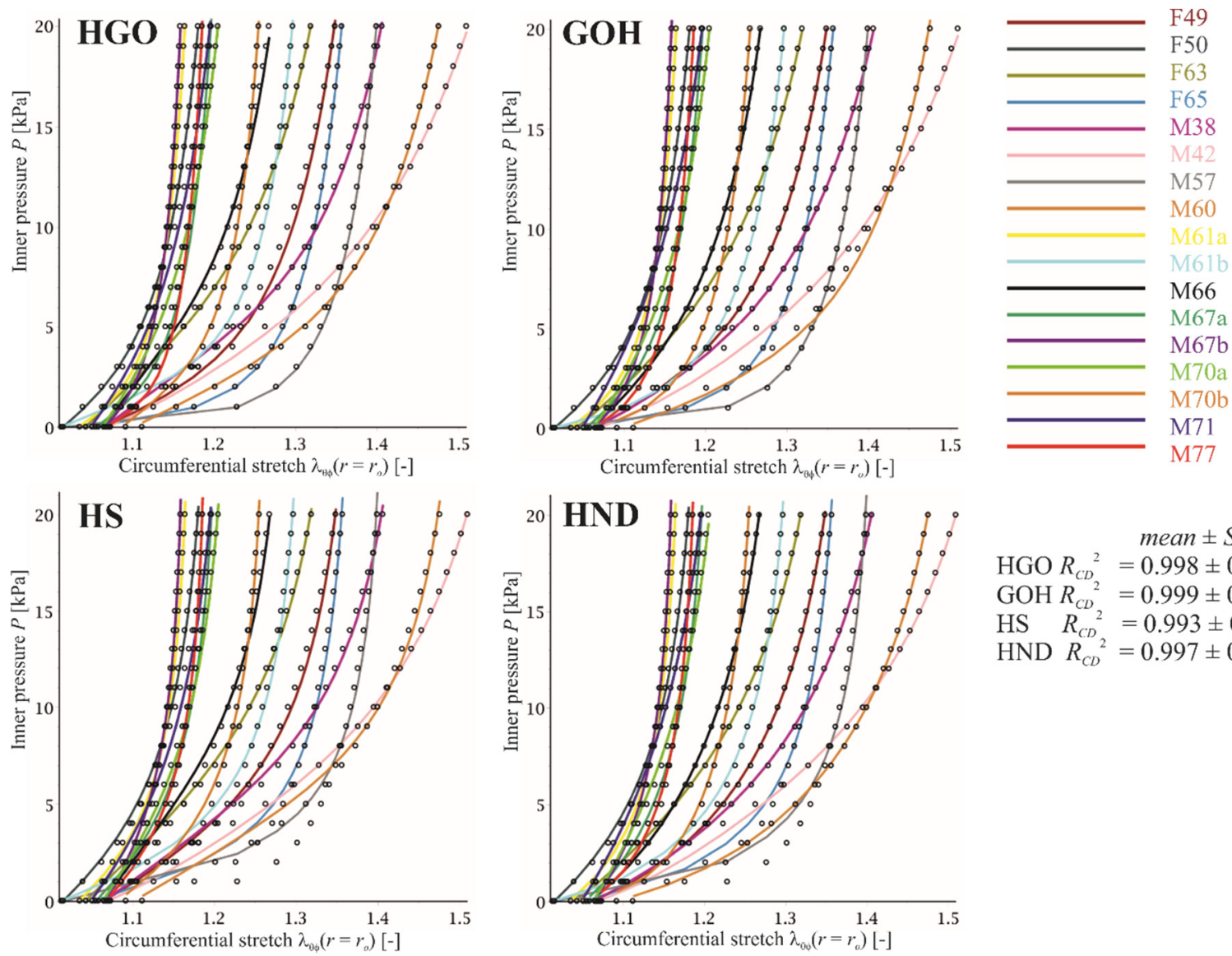

$$
\begin{array}{lc} 
& \text { mean } \pm S D \\
\text { HGO } R_{C D}{ }^{2}= & 0.998 \pm 0.003 \\
\text { GOH } R_{C D}{ }^{2}= & 0.999 \pm 0.001 \\
\text { HS } R_{C D}{ }^{2}= & 0.993 \pm 0.005 \\
\text { HND } R_{C D}{ }^{2}=0.997 \pm 0.004
\end{array}
$$

Figure 2. Inner pressure predicted by structurally-motivated models $W_{H G O}, W_{G O H}, W_{H S}, W_{H N D}$ and simulated by $W_{G M W}$. Points represent inflation-extension data obtained from simulation based on Labrosse et al. (2013). Although all models mutually correspond well (see coefficients of determination in Tables 2, 3, 4 and 5), one can see that limiting extensibility models somewhat overestimate pressures at infra-physiological loading. This is the case in particular of F65, M57, M67b, and M77.

\subsection{Estimated material parameters}

Material parameters for structurally-motivated constitutive models obtained in the nonlinear regression are respectively listed for the $W_{H G O}$ model in Table 2, for $W_{G O H}$ in Table 3, for $W_{H S}$ in Table 4, and for $W_{H N D}$ in Table 5. Mean values for the set of 17 aortas are quoted either as an average or a median depending on whether the data follows normal distribution. Similarly, variability is characterised either with sample standard deviation (SD) or by lower and upper quartile.

Figure 2 depicts inflation-extension response of abdominal aortas (points) and predictions given by studied models. All models fit the response well. There are some cases where $R_{C D}$ reached values higher than 0.9999 . Comparing models by $R_{C D}{ }^{2}$, one finds that the best fits are obtained by $W_{G O H}$ and that exponential models are slightly better than limiting extensibility ones. Especially in F65, M57, M67b, and M77, limiting extensibility models somewhat overestimate pressures in infra-physiological conditions. However, it has to be pointed out that none of the presented models give a prediction worse than $R_{C D}{ }^{2}=0.99$.

Figure 3 depicts unbalanced force predicted by models $W_{H G O}, W_{G O H}, W_{H S}$ and $W_{H N D}$. It is better to say this is the difference from the condition $F_{r e d}=0$ which was prescribed in the nonlinear regression. 
As expressed in the integral (17), $F_{\text {red }}$ is the force acting on closed ends of the tube additionally to the pressure generated force. Labrosse et al. (2013) conducted their experiments without additional axial prestretching, thus the satisfaction of this condition is another criterion of the quality of a model. Zero "observed" force, however, implies singularity in $R_{C D}^{2}$ computed for the force. In such a case, one could hypothesise that the lower the deviation from zero, the better the model is.

From Figure 3, it is clear that exponential models are better than limiting extensibility ones and that introduction of fibre dispersion into the strain energy density significantly decreases deviation of the force from zero.
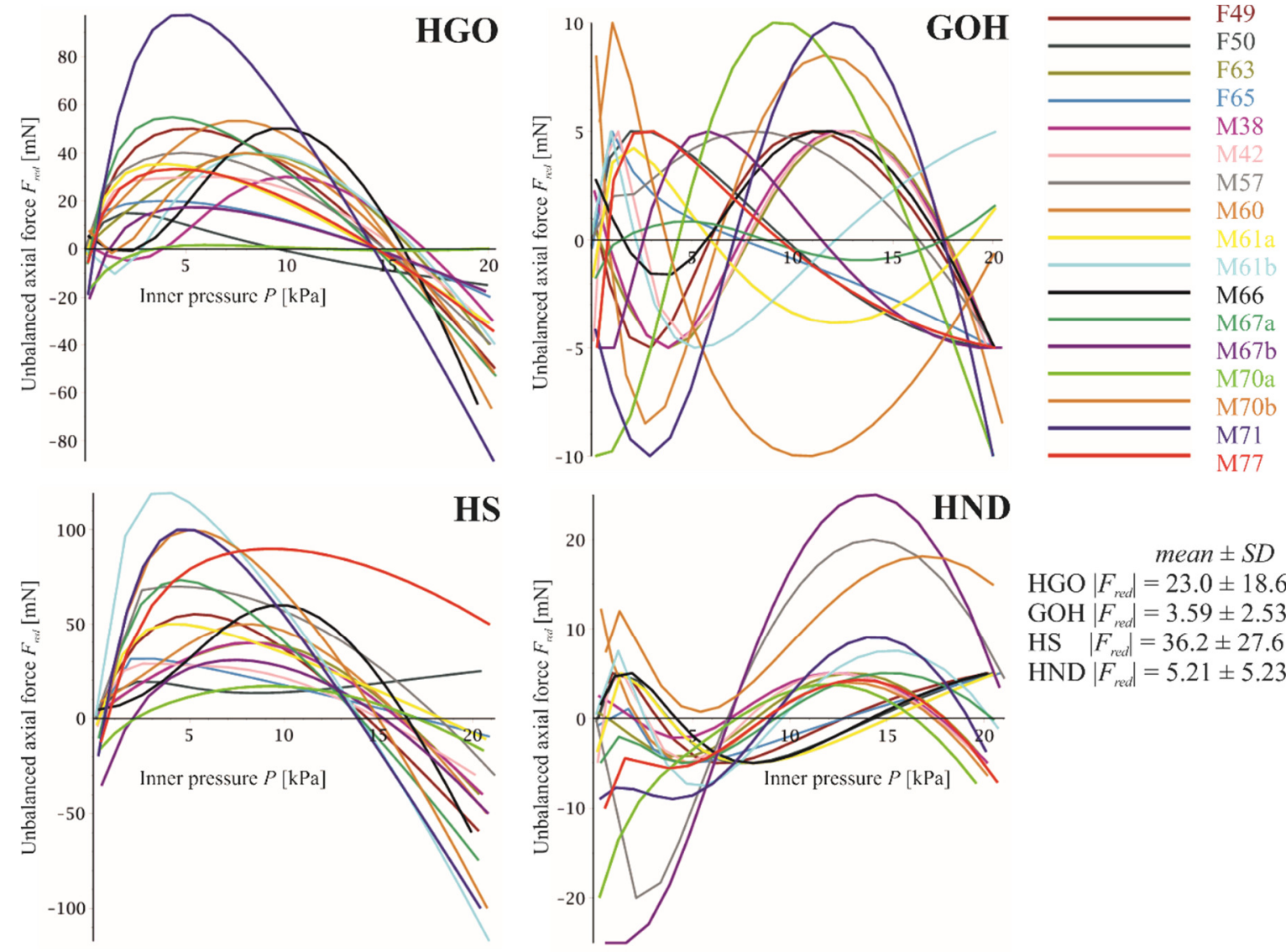

mean $\pm S D$

HGO $\left|F_{\text {red }}\right|=23.0 \pm 18.6 \mathrm{mN}$ GOH $\left|F_{\text {red }}\right|=3.59 \pm 2.53 \mathrm{mN}$ HS $\left|F_{\text {red }}\right|=36.2 \pm 27.6 \mathrm{mN}$ HND $\left|F_{\text {red }}\right|=5.21 \pm 5.23 \mathrm{mN}$

Figure 3. Unbalanced axial force predicted by models. Axial force, $F_{\text {red }}$, acting on the ends of tube additionally to the force generated by internal pressure acting on the cross-section of the tube's plugs, is prescribed to be zero. This condition was, however, not satisfied perfectly in nonlinear regression. Clearly, addition of the dispersion of fibres into the models of the strain energy density improved predictions about one order of the magnitude. Nevertheless, it is worth noting that the force of $100 \mathrm{mN}$ corresponds to the weight of $10 \mathrm{~g}$ which in true experiment (consider vertical configuration of pressurised tube) could be more that the mass of the sample itself which effect is usually neglected.

\subsection{Correlation with age}

The results of correlation analysis are presented in Table 6 . None of the treated models reached significant correlation between age and $\mu$ representing isotropic matrix $\left(\left|R_{L C}\right|<0.25\right.$ and thus is far from significance considering that the number of samples is 17). The correlation coefficient between age and the orientation of preferred directions $\beta$ is negative but also did not reach statistical significance. Also insignificant is the correlation obtained for fibre dispersion characterised by $\kappa$. 
Horný L, Netušil M, Daniel M. (2014) Limiting extensibility constitutive model with distributed fibre orientations and ageing of abdominal aorta. Journal of the Mechanical Behavior of Biomedical Materials, 38:39-51. DOI: 10.1016/j.jmbbm.2014.05.021 MANUSCRIPT VERSION http://dx.doi.org/10.1016/j.jmbbm.2014.05.021

Table 2. Material parameters of $W_{H G O}$. In the last column, descriptive statistics of location and dispersion for data set of all samples are listed. There are two rows in case that Shapiro-Wilk test confirmed normality (mean \pm sample standard deviation). In case that data does not follow normal distribution, there are three rows respectively expressing median, lower quartile, and upper quartile. The line entitled $\left|F_{\text {red }}\right|$ expresses descriptive statistics of obtained absolute values of the reduced axial force for 21 pressures used in the regression. The last cell is computed for the set of $17 \times 21$ forces.

\begin{tabular}{|c|c|c|c|c|c|c|c|c|c|c|c|c|c|c|c|c|c|c|}
\hline ID & F49 & F50 & F63 & F65 & M38 & M42 & M57 & M60 & M61a & M61b & M66 & M67a & M67b & M70a & M70b & M71 & M77 & $\begin{array}{c}\text { Location } \\
\text { Dispersion }\end{array}$ \\
\hline$\mu[\mathrm{kPa}]$ & 4.621 & 1.357 & & & 24.39 & & & & & & & & & & & & & \\
\hline$k_{1}[\mathrm{k}$ & 0.06 & 49.54 & 41.78 & .063 & 19.28 & 21.88 & 4.371 & 17.57 & 49.45 & 11.37 & 25.72 & 41.95 & 61.02 & 67.45 & 17.31 & 94.49 & 26.65 & \\
\hline$k_{2}[-]$ & & 7.438 & 3.286 & 5.869 & 3.216 & & & 3.178 & 26.74 & 8.045 & 7.647 & 22.00 & 38.36 & 7.022 & 22.86 & 9.262 & 39.30 & \\
\hline$\beta\left[^{\circ}\right]$ & 9.95 & 36.67 & 39.86 & 39.36 & 41.60 & 38.34 & 41.11 & 43.16 & 37.55 & 41.95 & 38.85 & 38.18 & 37.05 & 35.49 & 39.69 & 37.65 & 38.23 & \\
\hline$R_{C D}{ }^{2}[-]$ & 0.9929 & 1.000 & 1.000 & 0.9999 & 0.9994 & 0.9980 & 0.9995 & 0.9997 & 1.000 & 0.9980 & 0.9960 & 0.9925 & 0.9994 & 0.9998 & 0.9987 & 0.9917 & 0.9944 & \\
\hline $\begin{array}{l}\text { red }[\mathrm{mN}] \\
\text { spersion }\end{array}$ & $\begin{array}{c}30.79 \\
\pm 16.58\end{array}$ & $\begin{array}{c}8.270 \\
\pm 4.934\end{array}$ & $\begin{array}{r}24.26 \\
\pm 13.12\end{array}$ & $\begin{array}{l}14.30 \\
6.517 \\
18.74 \\
\end{array}$ & $\begin{array}{l}16.08 \\
4.201 \\
26.71 \\
\end{array}$ & $\begin{array}{l}22.94 \\
11.73 \\
29.01 \\
\end{array}$ & $\begin{array}{l}28.24 \\
14.20 \\
37.37 \\
\end{array}$ & $\begin{array}{c}34.48 \\
\pm 17.92 \\
\end{array}$ & $\begin{array}{r}20.50 \\
\pm 11.47 \\
\end{array}$ & $\begin{array}{c}22.93 \\
\pm 13.90 \\
\end{array}$ & $\begin{array}{c}28.30 \\
\pm 19.70\end{array}$ & $\begin{array}{c}33.14 \\
\pm 17.74 \\
\end{array}$ & & $\begin{array}{c}0.6554 \\
0.3287 \\
1.490 \\
\end{array}$ & & & & \\
\hline
\end{tabular}

Table 3. Material parameters of $W_{G O H}$. The table is arranged in the same way as Table 2 .

\begin{tabular}{|c|c|c|c|c|c|c|c|c|c|c|c|c|c|c|c|c|c|c|}
\hline ID & F49 & F50 & F63 & F65 & M38 & M42 & M57 & M60 & M61a & M61b & M66 & M67a & M67b & M70a & M70b & M71 & M77 & $\begin{array}{c}\text { Location } \\
\text { Dispersion } \\
\end{array}$ \\
\hline$\mu[\mathrm{kPa}]$ & 11.55 & 8.112 & 8.78 & & 15.90 & 0.2309 & 0.1 & 10.67 & 9.065 & 5.247 & 20.68 & 15.81 & 23.24 & 18.91 & 1.399 & 40.29 & 2.651 & \\
\hline$k_{1}[\mathrm{kPa}]$ & 63.05 & 76.69 & 113.8 & 17.76 & 78.49 & 29.66 & 14.94 & 55.98 & 166.9 & 100.5 & 116.5 & 209.0 & 325.0 & 123.4 & 310.9 & 253.1 & 153.8 & \\
\hline & & & & & & & & 7.777 & & & & 66.36 & 192.3 & & & & & \\
\hline & 38.18 & 35.16 & 38.99 & 38.39 & 41.41 & 37.39 & 39.73 & 44.36 & 35.95 & 42.83 & 38.39 & 36.42 & 33.11 & 32.18 & 39.60 & 34.60 & 37.31 & \\
\hline$\kappa[-]$ & 0.1874 & 0.0956 & 0.1688 & 0.1115 & 0.1875 & 0.0718 & 0.1819 & 0.2016 & 0.1797 & 0.2460 & 0.1820 & 0.2074 & 0.2301 & 0.1265 & & 0.179 & 0.1935 & $\begin{array}{r}0.1 \\
\pm 0.0\end{array}$ \\
\hline$R_{C D}{ }^{2}[-]$ & 9999 & 1.0 & 0.9 & & 0.99 & & & & & 0.9 & 0.9999 & & 0.9987 & & 0.9998 & 0.995 & 0.9998 & \\
\hline $\begin{array}{l}\text { red }[\mathrm{mN}] \\
\text { ispersion }\end{array}$ & $\begin{array}{r}3.07 \\
\pm 1.6\end{array}$ & $\begin{array}{l}3.584 \\
1.666 \\
4.568\end{array}$ & $\begin{array}{l}3.227 \\
1.426 \\
4.577\end{array}$ & $\begin{array}{c}2.153 \\
\pm 1.595\end{array}$ & $\begin{array}{l}2.675 \\
1.901 \\
4.566\end{array}$ & $\begin{array}{l}3.558 \\
2.074 \\
4.674\end{array}$ & $\begin{array}{c}3.134 \\
\pm 1.595\end{array}$ & $\begin{array}{l}6.143 \\
3.048 \\
8.183\end{array}$ & $\begin{array}{c}2.429 \\
\pm 1.262\end{array}$ & $\begin{array}{l}2.974 \\
1.326 \\
4.545\end{array}$ & $\begin{array}{l}2.810 \\
1.073 \\
4.257\end{array}$ & $\begin{array}{l}0.6487 \\
0.2963 \\
0.8548\end{array}$ & $\begin{array}{l}3.893 \\
1.906 \\
4.873\end{array}$ & $\begin{array}{l}7.299 \\
3.988 \\
9.501\end{array}$ & $\begin{array}{c}6.524 \\
\pm 3.083\end{array}$ & $\begin{array}{c}6.497 \\
\pm 3.101\end{array}$ & & $\begin{array}{l}3.483 \\
1.464 \\
4.964\end{array}$ \\
\hline
\end{tabular}

Table 4. Material parameters of $W_{H S}$. The table is arranged in the same way as Table 2.

\begin{tabular}{|c|c|c|c|c|c|c|c|c|c|c|c|c|c|c|c|c|c|c|}
\hline ID & F49 & F50 & F63 & F65 & M38 & M42 & M57 & M60 & M61a & M61b & M66 & M67a & M67b & M70a & M70b & M71 & M77 & $\begin{array}{c}\text { Location } \\
\text { Dispersion } \\
\end{array}$ \\
\hline$\mu[\mathrm{kPa}]$ & 7.463 & 0.1 & 28.65 & 0.1 & 18.84 & 0.1 & 0.1 & 15.16 & 0.1 & 2.029 & 32.41 & 0.1 & 0.1 & 6.989 & 0.6294 & 0.1 & 0.1 & $\begin{array}{c}0.1 \\
0.1 \\
10.03\end{array}$ \\
\hline$v[\mathrm{kPa}]$ & 26.33 & 61.48 & 45.51 & 20.36 & 27.69 & 23.53 & 16.88 & 21.18 & 76.29 & 28.83 & 33.52 & 70.60 & 113.6 & 70.23 & 54.19 & 99.38 & 69.16 & $\begin{array}{c}50.52 \\
\pm 29.40\end{array}$ \\
\hline$J_{m}[-]$ & 0.5026 & 0.2460 & 0.4790 & 0.4986 & 0.6295 & 2.385 & 0.7709 & 0.6117 & 0.0868 & 0.3755 & 0.2453 & 0.1144 & 0.07372 & 0.2234 & 0.1482 & 0.16220 & 0.08373 & $\begin{array}{l}0.2460 \\
0.1370 \\
0.5390\end{array}$ \\
\hline$\beta\left[^{\circ}\right]$ & 39.85 & 36.84 & 39.84 & 39.40 & 41.61 & 38.36 & 41.18 & 43.30 & 37.61 & 41.78 & 38.98 & 38.16 & 36.98 & 35.43 & 39.66 & 37.65 & 38.43 & $\begin{array}{c}39.12 \\
\pm 2.034\end{array}$ \\
\hline$R_{C D}{ }^{2}[-]$ & 0.9954 & 0.9982 & 0.9990 & 0.9894 & 0.9975 & 0.9989 & 0.9794 & 0.9934 & 0.9944 & 0.9902 & 0.9938 & 0.9958 & 0.9913 & 0.9953 & 0.9910 & 0.9850 & 0.9889 & $\begin{array}{c}0.9928 \\
\pm 5.189 \mathrm{E}-3\end{array}$ \\
\hline $\begin{array}{l}\left|F_{\text {red }}\right|[\mathrm{mN}] \\
\text { Dispersion }\end{array}$ & $\begin{array}{c}34.18 \\
\pm 18.65\end{array}$ & $\begin{array}{l}15.95 \\
14.12 \\
19.36\end{array}$ & $\begin{array}{c}24.87 \\
\pm 13.05\end{array}$ & $\begin{array}{c}16.14 \\
\pm 10.56\end{array}$ & $\begin{array}{c}24.81 \\
\pm 12.90\end{array}$ & $\begin{array}{l}21.17 \\
10.31 \\
27.12\end{array}$ & $\begin{array}{l}54.87 \\
28.48 \\
67.61\end{array}$ & $\begin{array}{c}31.95 \\
\pm 15.58\end{array}$ & $\begin{array}{c}28.30 \\
\pm 16.95\end{array}$ & $\begin{array}{c}67.91 \\
\pm 39.98\end{array}$ & $\begin{array}{c}35.63 \\
\pm 20.40\end{array}$ & $\begin{array}{c}43.40 \\
\pm 23.77\end{array}$ & $\begin{array}{c}22.56 \\
\pm 11.82\end{array}$ & $\begin{array}{l}12.06 \\
7.170 \\
16.36 \\
\end{array}$ & $\begin{array}{l}68.48 \\
33.06 \\
92.19\end{array}$ & $\begin{array}{c}59.08 \\
\pm 32.38\end{array}$ & $\begin{array}{l}81.07 \\
64.50 \\
88.21 \\
\end{array}$ & $\begin{array}{l}28.87 \\
15.07 \\
51.36 \\
\end{array}$ \\
\hline
\end{tabular}


Horný L, Netušil M, Daniel M. (2014) Limiting extensibility constitutive model with distributed fibre orientations and ageing of abdominal aorta. Journal of the Mechanical Behavior of Biomedical Materials, 38:39-51. DOI: 10.1016/j.jmbbm.2014.05.021 MANUSCRIPT VERSION http://dx.doi.org/10.1016/j.jmbbm.2014.05.021

Table 5. Material parameters of $W_{H N D}$. The table is arranged in the same way as Table 2.

\begin{tabular}{|c|c|c|c|c|c|c|c|c|c|c|c|c|c|c|c|c|c|c|}
\hline ID & F49 & F50 & F63 & F65 & M38 & M42 & M57 & M60 & M61a & M61b & M66 & M67a & M67b & M70a & M70b & M71 & M77 & $\begin{array}{c}\text { Location } \\
\text { Dispersion }\end{array}$ \\
\hline$\mu[\mathrm{kPa}]$ & 4.884 & 3.456 & 28.04 & 0.1 & 15.04 & 0.2514 & 0.1 & 8.518 & 1.952 & 5.838 & 14.40 & 12.19 & 11.94 & 8.561 & 0.1 & 32.28 & 0.1 & $\begin{array}{c}5.838 \\
0.2010 \\
12.92\end{array}$ \\
\hline$v[\mathrm{kPa}]$ & 85.01 & 83.81 & 123.9 & 41.25 & 88.42 & 31.52 & 57.18 & 109.8 & 223.1 & 143.3 & 169.2 & 289.8 & 405.4 & 90.41 & 484.4 & 268.0 & 372.0 & $\begin{array}{l}123.9 \\
84.61 \\
275.3\end{array}$ \\
\hline$J_{m}[-]$ & 0.2314 & 0.1794 & 0.2371 & 0.2601 & 0.2987 & 1.971 & 0.2464 & $0.2155 \mathrm{C}$ & 0.03196 & 0.08530 & 0.09758 & 0.02917 & 0.02123 & 0.2422 & 0.02236 & 0.07478 & 0.01801 & $\begin{array}{c}0.1794 \\
0.03103 \\
0.2436\end{array}$ \\
\hline$\beta\left[^{\circ}\right]$ & 38.51 & 35.87 & 38.99 & 37.92 & 41.40 & 37.37 & 38.37 & 45.06 & 36.38 & 43.01 & 38.36 & 36.47 & 34.87 & 35.01 & 39.68 & 35.18 & 36.93 & $\begin{array}{c}38.20 \\
\pm 2.289\end{array}$ \\
\hline$\kappa[-]$ & 0.1872 & 0.0691 & 0.1711 & 0.1440 & 0.1906 & 0.0728 & 0.2371 & 0.2421 & 0.1657 & 0.2536 & 0.2001 & 0.2113 & 0.1910 & 0.0230 & 0.2677 & 0.1703 & 0.2234 & $\begin{array}{c}0.1777 \\
\pm 0.06791\end{array}$ \\
\hline$R_{C D}^{2}[-]$ & 0.9995 & 0.9983 & 0.9997 & 0.9916 & 0.9996 & 0.9983 & 0.9851 & 0.9995 & 0.9961 & 0.9951 & 0.9998 & 0.9976 & 0.9924 & 0.9968 & 0.9976 & 0.9999 & 0.9935 & $\begin{array}{l}0.9976 \\
0.9946 \\
0.9996\end{array}$ \\
\hline $\begin{array}{l}\left|F_{\text {red }}\right|[\mathrm{mN}] \\
\text { Dispersion }\end{array}$ & $\begin{array}{l}3.284 \\
1.513 \\
4.438\end{array}$ & $\begin{array}{l}3.370 \\
1.526 \\
4.560\end{array}$ & $\begin{array}{l}3.327 \\
1.734 \\
4.265\end{array}$ & $\begin{array}{c}2.767 \\
\pm 1.620\end{array}$ & $\begin{array}{c}2.623 \\
\pm 1.615\end{array}$ & $\begin{array}{l}3.639 \\
2.180 \\
4.948\end{array}$ & $\begin{array}{l}13.76 \\
6.000 \\
18.41\end{array}$ & $\begin{array}{l}3.279 \\
1.858 \\
4.313\end{array}$ & $\begin{array}{c}3.175 \\
\pm 1.597\end{array}$ & $\begin{array}{l}5.166 \\
2.131 \\
7.024\end{array}$ & $\begin{array}{r}3.086 \\
\pm 1.611\end{array}$ & $\begin{array}{l}3.581 \\
2.056 \\
4.712\end{array}$ & $\begin{array}{l}18.50 \\
10.81 \\
23.87\end{array}$ & $\begin{array}{l}3.220 \\
1.987 \\
23.87\end{array}$ & $\begin{array}{c}9.974 \\
\pm 6.187\end{array}$ & $\begin{array}{l}7.266 \\
3.920 \\
8.595\end{array}$ & $\begin{array}{c}3.694 \\
\pm 2.287\end{array}$ & $\begin{array}{l}3.872 \\
2.062 \\
5.042\end{array}$ \\
\hline
\end{tabular}

The parameters scaling nonlinear functions in the models $\left(k_{1}\right.$ and $\left.v\right)$ significantly increase in the linear statistical model with advancing age except $W_{H G O}\left(W_{H G O}: R_{L C}=0.346, p\right.$ value $=0.174 ; W_{G O H}$ : $R_{L C}=0.570, p$ value $=0.018 ; W_{H S}: R_{L C}=0.531, p$ value $=0.033 ; W_{H N D}: R_{L C}=0.636, p$ value $\left.=0.006\right)$. Nonlinear coefficients, $k_{2}$ governing abruptness of the large strain stiffening and $J_{m}$ expressing limiting extensibility, reached statistical significance in all models where they are present. Due to their different mathematical nature, $k_{2}$ increases with age $\left(W_{H G O}: R_{L C}=0.577, p\right.$ value $=0.016 ; W_{G O H}: R_{L C}=0.482, p$ value $=0.05)$, whereas $J_{m}$ decreases $\left(W_{H S}: R_{L C}=-0.640, p\right.$ value $=0.006 ; W_{H N D}: R_{L C}=-0.567, p$ value $=0.018)$.

Table 7 contains parameters of elementary functions used to describe age-related changes of constitutive parameters $k_{1}, k_{2}, v$ and $J_{m}$. The best results were in all cases obtained by $a^{b x}$, where $x$ stands for age in years. Figure 4 displays the evolution of limiting extensibility parameter $J_{m}$ in ageing. Although $J_{m}$ is the constitutive parameter, the geometrically relevant information is in the expression $J_{m}{ }^{1 / 2}+1$. Its evolution is thus depicted rather than $J_{m}$.

Table 6. Correlation between age and constitutive parameters. Bold typesetting indicates statistical significance at the level 0.05 .

\begin{tabular}{ccccccc} 
Model & \multicolumn{5}{c}{ Parameters } \\
\hline \hline$W_{G M W}$ & $c_{1}$ & $\boldsymbol{c}_{2}$ & $\boldsymbol{c}_{3}$ & & \\
$R_{L C}$ & -0.469 & $\mathbf{0 . 5 1 1}$ & $\mathbf{0 . 5 9 6}$ & & \\
$p$ value & 0.058 & 0.038 & 0.012 & & \\
$W_{H G O}$ & $\mu$ & $k_{1}$ & $\boldsymbol{k}_{\mathbf{2}}$ & $\beta$ \\
$R_{L C}$ & -0.059 & 0.346 & $\mathbf{0 . 5 7 7}$ & -0.319 \\
p value & 0.820 & 0.174 & 0.016 & 0.212 & \\
$W_{G O H}$ & $\mu$ & $\boldsymbol{k}_{\mathbf{1}}$ & $\boldsymbol{k}_{\mathbf{2}}$ & $\beta$ & $\kappa$ \\
$R_{\text {LC }}$ & 0.235 & $\mathbf{0 . 5 7 0}$ & $\mathbf{0 . 4 8 2}$ & -0.305 & 0.384 \\
$p$ value & 0.364 & 0.018 & 0.05 & 0.234 & 0.129 \\
$W_{H S}$ & $\mu$ & $\boldsymbol{v}$ & $\boldsymbol{J}_{\boldsymbol{m}}$ & $\beta$ & \\
$R_{\text {LC }}$ & -0.127 & $\mathbf{0 . 5 3 1}$ & $\mathbf{- 0 . 6 4 0}$ & -0.317 & \\
$p$ value & 0.638 & 0.033 & 0.006 & 0.230 & \\
$W_{\text {HND }}$ & $\mu$ & $\boldsymbol{v}$ & $\boldsymbol{J}_{\boldsymbol{m}}$ & $\beta$ & $\kappa$ \\
$R_{L C}$ & 0.150 & $\mathbf{0 . 6 3 6}$ & $\mathbf{- 0 . 5 6 7}$ & -0.288 & 0.242 \\
p value & 0.568 & 0.006 & 0.018 & 0.262 & 0.350 \\
\hline \multicolumn{7}{c}{}
\end{tabular}



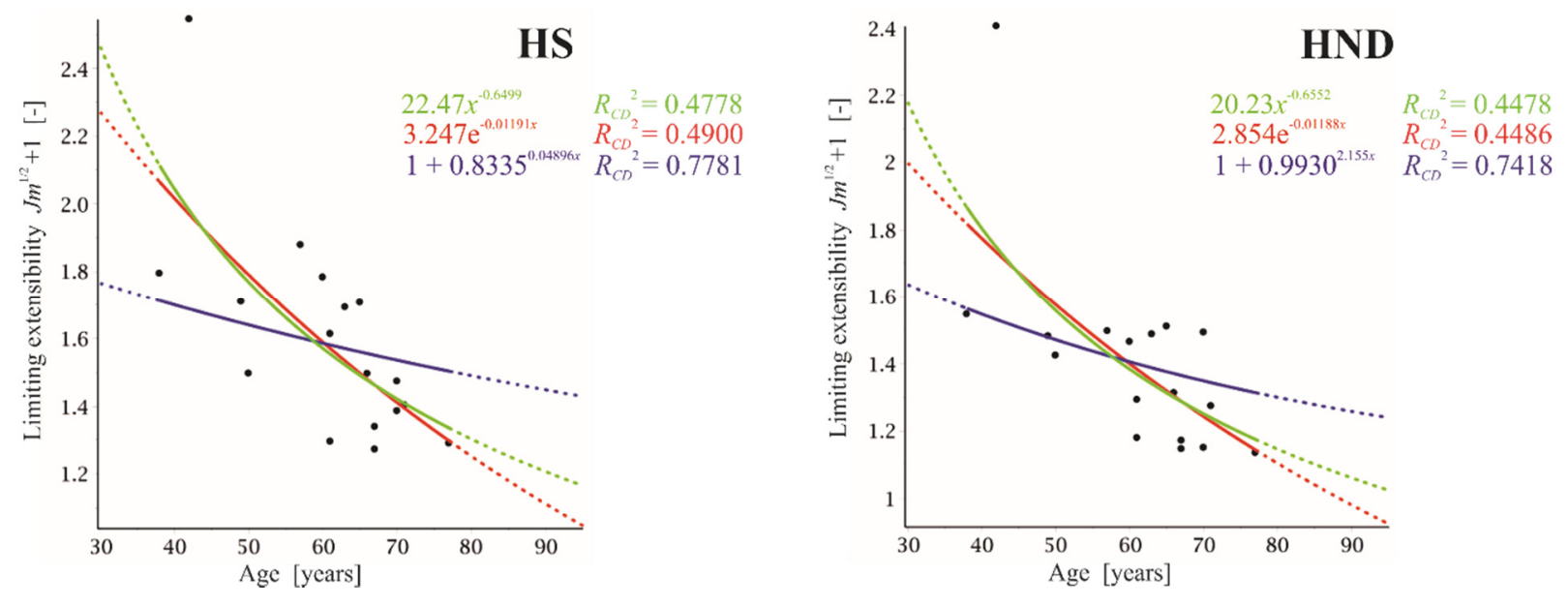

Figure 4. Evolution of limiting extensibility in ageing. Left panel displays $J_{m}^{1 / 2}+1$ obtained with $W_{H S}$ and right one in $W_{H N D}$. Solid regression curves are used in the interval from 38 to 77 years whereas dotted curves indicate the area of extrapolation. Note that $J_{m}^{1 / 2}+1$ showed the highest linear correlation with age: $R_{L C}=-0.7032$ with $p$ value $<0.001$ for $W_{H S}$, and $R_{L C}=-0.6687$ with $p$ value $<0.002$ for $W_{H N D}$.

\section{DISCUSSION AND CONCLUSION}

The objective of the present study was to numerically characterise microstructurally-motivated constitutive models of the human abdominal aorta suitable for description of mechanical response under a multiaxial state of stress (inflation-extension of the thick-walled tube). Four different anisotropic hyperelastic constitutive models were used. Two invariant-based exponential-type strain energy density functions ( $W_{H G O}$ - Holzapfel et al., 2000; $W_{G O H}$ - Gasser et al., 2006) were employed because they are probably the most frequently used in computational biomechanics at present. Horny et al. (2013a) have recently shown the concept of limiting extensibility is suitable to be used in the description of age-related changes in the mechanical behaviour of human arteries. Hence, $W_{H G O}$ and $W_{G O H}$ were supplemented by two limiting fibre extensibility models. Particularly, it was the strain energy suggested by Horgan and Saccomandi (2005), $W_{H S}$, and the second one is used for the first time in our study, $W_{H N D}$, and combines the idea of finitely extensible reinforcing fibres with a dispersion of fibre orientations.

The models were calibrated to an inflation-extension response obtained in the simulation which adopted material parameters and geometries published by Labrosse et al. (2013) who, however, used a purely phenomenological strain energy density function of the Fung-type, $W_{G M W}$. The results of the simulation were used in the model calibration instead of the experimental data because Labrosse et al. (2013) did not include experimental curves into their paper. The simulation as well as the subsequent regression analysis were based on the theory of the residually-stressed nonlinearly-elastic, anisotropic thick-walled tube. The coefficient of determination confirmed that all used models predict inflation pressures well $\left(R_{C D}^{2}>0.99\right.$ in all cases).

The present study delivers to readers three main points. The first is that anisotropic limiting extensibility models are suitable to describe the inflation-extension behaviour of the human aorta. For this reason, they provide an alternative to the models based on exponential function capturing large strain stiffening ascribed to the engagement of collagen fibres to a load-bearing process which are predominantly used in biomechanics. To the best of our knowledge, this is the first study presenting specific numerical values of limiting extensibility models for human arteries obtained at 3D stress states.

For the sake of completeness, it has to be said, however, that in the direct comparison of $W_{H G O}$ and $W_{H S}$, and $W_{G O H}$ and $W_{H N D}$, which create pairs of the strain energies mutually corresponding in the mathematical handling of anisotropy, exponential models satisfied both predictions of the internal 
pressure and zero reduced axial force slightly better than limiting extensibility models. The mean values for $R_{C D}{ }^{2}$ are 0.9994 for $W_{H G O}$ and 0.9928 for $W_{H S}$. In case of the models with fibre dispersion, $R_{C D}{ }^{2}=0.9998$ for $W_{G O H}$, and $R_{C D}{ }^{2}=0.9976$ for $W_{H N D}$ were obtained. The same conclusion can be derived from Figure 3 displaying deviation from the condition $F_{r e d}=0$. Comparing Figure 2 and Figure 3, it is also clear how the fibre dispersion contributed to the description of mechanical response. Its inclusion led to significant improvement of the satisfaction $F_{\text {red }}=0$. On the other hand, slightly better fits obtained by exponential-type models are not surprising when one considers that the data generated by another exponential-type model serves as an etalon.

Here it is worth noting that theoretically it could be better to formulate (21) as a single-valued objective function in the form $Q^{R E G}=w_{P} \Sigma\left(P^{E X P}-P^{M O D}\right)^{2}+w_{F} \Sigma\left(F_{\text {red }}^{M O D}\right)^{2}$ where $w_{P}$ and $w_{F}$ are some weights. In such a case, no constraining inequality would be necessary. In fact, it was our first choice but the problems with appropriately estimating of $w_{F}$ were encountered in some aortas and we were not able to effectively solve them with the available software. This is why (21) was formulated as a constrained optimisation. The constrained optimisation, however, led to reduced axial force which, at the first sight, could appear rather large. It will be useful to estimate the magnitude of axial forces acting in a closed pressurised tube and compare them with what here we call unbalanced force (deviation from the condition $F_{\text {red }}=0$; Figure 3). Consider, for instance, that when one conducts an inflation-extension experiment with a sample of the human abdominal aorta in a vertical configuration (the sample hanging from a stand) and compute the gravity force of the sample itself. Using mean middle radius $7.73 \mathrm{~mm}$ (Table 1), mean thickness $1.42 \mathrm{~mm}$ (Table 1), minimal length of the sample as $50 \mathrm{~mm}$ and $0.001 \mathrm{gmm}^{-3}$ as the lower bound for the density of the artery wall, the obtained gravity force is approximately $34 \mathrm{mN}$ which is higher than the highest mean value of IFredl obtained in our study (it is $29 \mathrm{mN}$ for $W_{H S}$ ). Gravity force of the sample itself is, however, usually neglected. Moreover, considering axial force intrinsically present in the problem, the force generated the pressure at closed ends of the tube, for the same middle radius and thickness (disregarding of the deformation) as above, at physiological pressure $13.3 \mathrm{kPa}$ one obtains the force of approx. $916 \mathrm{mN}$. The existence of this force is captured by the model and differences from condition $F_{\text {red }}=0$ are lower than this force at about one order of the magnitude. This leads us to consider the imperfect predictions of axial force to be acceptable.

The second point is that the mean orientation of reinforcing fibres, or more precisely the orientation of preferred directions, does not change with age. Correlation coefficients in Table 6 support this conclusion. Although based on the coefficients themselves, one could expect a slight decrease of the inclination from circumferential axis during ageing $\left(-0.319<R_{L C}<-0.288\right), p$ values suggest we cannot reject the random effects against age-related trend $(0.212<p$ value $<0.262)$. Our conclusion is supported by results known from the literature (Table 1 in Roccabianca et al., 2014; Table 4 in Hasskett et al., 2010; Table 4 in Weisbecker et al., 2012). The data also suggest there is no statistically significant age-related trend in the fibre dispersion expressed with $\kappa$. Here we would like to point out that the Two-sample t-test and Two-sample paired t-test were performed for $\beta$ obtained by $W_{H G O}$ and $W_{G O H}$, and also for $W_{H S}$ and $W_{H N D}$. The results showed that although mean values of $\beta$ in $W_{H G O}$ and $W_{G O H}$ samples as such differ insignificantly from a statistical point of view, when pairs related to specific donor are considered, the mean value of $\beta$ in $W_{H G O}$ and in $W_{G O H}$ differ significantly (the same apply for $W_{H S}$ and $\left.W_{H N D}\right)$. This should not be a surprise, but it was conducted to make sure that the estimation of parameters leads to what one expects considering physical interpretation of the models. 
Table 7. Age-related changes in material parameters described by elementary functions. Symbol * indicates that in certain interval the model predicts negative values of the parameter. This is the consequence of the used time period (from 38 to 77 years) where the regression models minimize a sum of squares. Negativity would however lead to the loss of the convexity of $W$.

\begin{tabular}{|c|c|c|c|c|c|}
\hline$x=$ age [years] & $a \cdot x+b$ & $a \cdot x^{b}$ & $a \cdot \mathrm{e}^{b \cdot x}$ & $a \cdot \ln (\mathrm{x})+\mathrm{b}$ & $a^{b x}$ \\
\hline \multirow[t]{2}{*}{$W_{H G O} k_{1}[\mathrm{kPa}]$} & $a=0.7875$ & $a=0.3800$ & $a=7.649$ & $a=41.83$ & $a=1.019$ \\
\hline & $b=-$ & $b=1.035$ & $b=2.027 \mathrm{E}-2$ & -137.1 & $b=2.843$ \\
\hline$R_{C D}^{2}[-]$ & 0.1198 & 0.0001 & 0.01 & 0.1110 & 0.9647 \\
\hline \multirow{3}{*}{$\begin{array}{c}W_{H G O} k_{2}[-] \\
R_{C D}[-]\end{array}$} & $a=0.6748^{*}$ & $a=1.041 \mathrm{E}-6$ & $a=0.1028$ & $a=35.40^{*}$ & $a=1.016$ \\
\hline & $b=-28.46$ & $b=3.869$ & $b=7.119 \mathrm{E}-2$ & $b=-132.3$ & $b=2.630$ \\
\hline & 0.3333 & 0.2906 & 0.3378 & 0.3013 & 0.6545 \\
\hline \multirow{2}{*}{$\begin{array}{c}W_{G O H} k_{1}[\mathrm{kPa}] \\
R_{C D}{ }^{2}[-]\end{array}$} & $a=5.143$ & $a=8.323 \mathrm{E}-3$ & $a=6.604$ & $a=271.8^{*}$ & $a=1.023$ \\
\hline & $\begin{array}{c}b=-182.8 \\
0.3254\end{array}$ & $\begin{array}{c}b=2.282 \\
0.2127\end{array}$ & $b=4.373 \mathrm{E}-2$ & $b=-982.2$ & $b=3.210$ \\
\hline \multirow{3}{*}{$\begin{array}{c}W_{G O H} k_{2}[-] \\
R_{C D}[-]\end{array}$} & $a=2.351 *$ & $a=2.065 \mathrm{E}-8$ & $a=6.438 \mathrm{E}-2$ & $a=124.2 *$ & $a=1.020$ \\
\hline & $b=-104.2$ & $b=5.021$ & $b=9.193 \mathrm{E}-2$ & $b=-469.5$ & $b=2.978$ \\
\hline & 0.2327 & 0.1293 & 0.1557 & 0.2136 & 0.7482 \\
\hline \multirow{3}{*}{$\begin{array}{c}W_{H S} v[\mathrm{kPa}] \\
R_{C D^{2}}[-]\end{array}$} & $a=1.468$ & $a=6.003 \mathrm{E}-2$ & $a=6.687$ & 77.72 & $a=1.021$ \\
\hline & $b=-38.78$ & $b=1.606$ & $b=3.052 \mathrm{E}-2$ & $b=-267.5$ & $b=2.964$ \\
\hline & 0.2816 & 0.2297 & 0.2536 & 0.2593 & 0.7916 \\
\hline \multirow{3}{*}{$\begin{array}{c}W_{H S} J_{m}[-] \\
R_{C D}{ }^{2}[-]\end{array}$} & $a=-3.280 \mathrm{E}-2^{*}$ & $a=2.925 \mathrm{E} 5$ & $a=12.86$ & $a=-1.825^{*}$ & $a=0.9937$ \\
\hline & $b=2.444$ & $b=-3.381$ & $b=-6.248 \mathrm{E}-2$ & $b=-7.918$ & $b=2.165$ \\
\hline & 0.4097 & 0.3392 & 0.3664 & 0.4167 & 0.8558 \\
\hline \multirow{3}{*}{$\begin{array}{c}W_{H N D} v[\mathrm{kPa}] \\
R_{C D}[-]\end{array}$} & $a=8.229^{*}$ & $a=2.557 \mathrm{E}-3$ & $a=6.508$ & $a=431.3 *$ & $a=1.024$ \\
\hline & $b=-320.1$ & $b=2.659$ & $b=4.993 \mathrm{E}-2$ & -1584 & $b=3.305$ \\
\hline & 0.4042 & 0.3480 & 0.3897 & 0.3648 & 0.5608 \\
\hline \multirow{2}{*}{$\begin{array}{c}W_{H N D} J_{m}[-] \\
R_{C D}[-]\end{array}$} & $a=-2.425 \mathrm{E}-2^{*}$ & $a=1.075 \mathrm{E} 7$ & $a=17.47$ & $a=-1.370^{*}$ & $a=0.9895$ \\
\hline & $\begin{array}{c}b=1.726 \\
0.3211\end{array}$ & $\begin{array}{c}b=-4.487 \\
0.2568\end{array}$ & $\begin{array}{c}b=-8.270 \mathrm{E}-2 \\
0.2792\end{array}$ & $\begin{array}{c}b=5.858 \\
0.3369\end{array}$ & $\begin{array}{c}b=2.156 \\
0.8476\end{array}$ \\
\hline
\end{tabular}

The last, and simultaneously, the main point is in Figure 4 and says that limiting extensibility is the concept which is, through parameter $J_{m}$, highly sensitive to age-related changes in the biomechanics of arterial wall. In other words, the maximum extensibility expressed by $J_{m}^{1 / 2}+1$ significantly decreases during ageing and found correlation coefficients are the highest ones obtained in our study $\left(R_{L C}=-0.7032\right.$ with $p$ value $<0.001$ for $W_{H S}$, and $R_{L C}=-0.6687$ with $p$ value $<0.002$ for $\left.W_{H N D}\right)$. This presented conclusion is in accordance with results obtained by Horny et al. (2013a), Horgan and Saccomandi (2003a), and Destrade et al. (2009) who adopted isotropic model $W_{G}$. Our study thus extends the conclusion from limiting chain extensibility used in uniaxial stress state to limiting fibre extensibility at multiaxial stress state of anisotropic material. Specific numerical values of $J_{m}$ obtained in our study correspond well to data known from the literature for human tissues although it has to be noted that only a limited number of studies employing this concept exist (Horgan and Saccomandi, 2003a; Destrade et al., 2009; Horny et al., 2013a). Horny et al. (2013a) summarised it into the inequality $0<J_{m}<2.5$ which is also satisfied by our results.

Finally, this study presents new sets of microstructurally-motivated material parameters for the human abdominal aorta which can be used in computational simulations devoted to the effect of mechanical environment on ageing, adaptation and remodelling of the artery wall. The authors also hope that the study will inspire further research focusing on, for instance, the mutual relation between the herein presented limiting extensibility parameters and the inter- and intra-constituent interactions in the tissue (intra-molecular, inter-molecular, and inter-fibrillar crosslinking) which would allow not only to describe but also to explain age-related changes in the constitutive behaviour.

\section{ACKNOWLEDGEMENT}

L.H. was supported by Czech Ministry of Health in project NT 13302. M.D. was supported by Technology Agency of the Czech Republic in project TA 01010185. M.N. was supported by the Faculty of Mathematics and Physics of Charles University in Prague in project no. 260098/2014. 


\section{REFERENCES}

Ambrosi, D., Ateshian, G. A., Arruda, E. M., Cowin, S. C., Dumais, J., Goriely, A., Garikipati, K., Holzapfel, G.A., Humphrey, J.D., et al. (2011). Perspectives on biological growth and remodeling. Journal of the Mechanics and Physics of Solids, 59(4), 863-883.

Bäck, M., Gasser, T. C., Michel, J. B., \& Caligiuri, G. (2013). Biomechanical factors in the biology of aortic wall and aortic valve diseases. Cardiovascular Research, 99(2), 232-241.

Corti, R., \& Fuster, V. (2003). New understanding, diagnosis, and prognosis of atherothrombosis and the role of imaging. American Journal of Cardiology, 91(3 SUPPL.), 17A-26A.

Chuong, C. J., \& Fung, Y. C. (1983). Three-dimensional stress distribution in arteries. Journal of Biomechanical Engineering, 105(3), 268-274.

Decraemer, W. F., Maes, M. A., \& Vanhuyse, V. J. (1980). An elastic stress-strain relation for soft biological tissues based on a structural model. Journal of Biomechanics, 13(6), 463-468.

Destrade, M., Ní Annaidh, A., \& Coman, C. D. (2009). Bending instabilities of soft biological tissues. International Journal of Solids and Structures, 46(25-26), 4322-4330.

Dougherty, G., \& Varro, J. (2000). A quantitative index for the measurement of the tortuosity of blood vessels. Medical Engineering and Physics, 22(8), 567-574.

Fung, Y. C. (1967). Elasticity of soft tissues in simple elongation. The American Journal of Physiology, 213(6), $1532-1544$

Fung, Y. C., Fronek, K., \& Patitucci, P. (1979). Pseudoelasticity of arteries and the choice of its mathematical expression. The American Journal of Physiology, 237(5), H620-631.

Fung, Y. C. (1997) Biomechanics: Circulation. Springer Science+Business Media, New York.

Gasser, T. C., Ogden, R. W., \& Holzapfel, G. A. (2006). Hyperelastic modelling of arterial layers with distributed collagen fibre orientations. Journal of the Royal Society Interface, 3(6), 15-35.

Gent, A. N. (1996). A new constitutive relation for rubber. Rubber Chemistry and Technology, 69(1), 59-61.

Greenwald, S. E. (2007). Ageing of the conduit arteries. Journal of Pathology, 211(2), 157-172.

Guccione, J. M., McCulloch, A. D., \& Waldman, L. K. (1991). Passive material properties of intact ventricular myocardium determined from a cylindrical model. Journal of Biomechanical Engineering, 113(1), 42-55.

Haskett, D., Johnson, G., Zhou, A., Utzinger, U., \& Vande Geest, J. (2010). Microstructural and biomechanical alterations of the human aorta as a function of age and location. Biomechanics and Modeling in Mechanobiology, 9(6), 725-736.

Holzapfel, G. A., Gasser, T. C., \& Ogden, R. W. (2000). A new constitutive framework for arterial wall mechanics and a comparative study of material models. Journal of Elasticity, 61(1-3), 1-48.

Holzapfel, G. A., \& Ogden, R. W. (2010). Constitutive modelling of arteries. Proceedings of the Royal Society A: Mathematical, Physical and Engineering Sciences, 466(2118), 1551-1597.

Horgan, C. O., \& Murphy, J. G. (2007). Limiting chain extensibility constitutive models of valanis-landel type. Journal of Elasticity, 86(2), 101-111.

Horgan, C. O., \& Saccomandi, G. (2002). A molecular-statistical basis for the gent constitutive model of rubber elasticity. Journal of Elasticity, 68(1-3), 167-176.

Horgan, C.O., \& Saccomandi, G. (2003a) A description of arterial wall mechanics using limiting chain extensibilty constitutive models. Biomechanics and Modeling in Mechanobiology, 1, 251-266.

Horgan, C. O., \& Saccomandi, G. (2003b). Finite thermoelasticity with limiting chain extensibility. Journal of the Mechanics and Physics of Solids, 51(6), 1127-1146.

Horgan, C. O., \& Saccomandi, G. (2005). A new constitutive theory for fiber-reinforced incompressible nonlinearly elastic solids. Journal of the Mechanics and Physics of Solids, 53(9), 1985-2015.

Horgan, C. O., \& Saccomandi, G. (2006). Phenomenological hyperelastic strain-stiffening constitutive models for rubber. Rubber Chemistry and Technology, 79(1), 152-169.

Horny, L., Hulan, M., Zitny, R., Chlup, H., Konvickova, S., \& Adamek, T. (2009). Computer-aided analysis of arterial wall architecture. IFMBE Proceedings, 25(4), 1494-1497.

Horny, L., Kronek, J., Chlup, H., Zitny, R., Vesely, J., \& Hulan, M. (2010). Orientations of collagen fibres in aortic histological sections. Bulletin of Applied Mecahnics, 6(22), 25-29.

Horny, L., Adamek, T., \& Zitny, R. (2013a). Age-related changes in longitudinal prestress in human abdominal aorta. Archive of Applied Mechanics, 83(6), 875-888.

Horný, L., Netušil, M., \& Voňavková, T. (2013b). Axial prestretch and circumferential distensibility in biomechanics of abdominal aorta. Biomechanics and Modeling in Mechanobiology, in press.

Humphrey, J. D. (1995). Mechanics of the arterial wall: Review and directions. Critical Reviews in Biomedical Engineering, 23(1-2), 1-162. 
Humphrey, J.D. (2002). Cardiovascular solid mechanics: Cells, tissues, organs. Springer-Verlag, New York.

Humphrey, J. D., \& Holzapfel, G. A. (2012). Mechanics, mechanobiology, and modeling of human abdominal aorta and aneurysms. Journal of Biomechanics, 45(5), 805-814.

Labrosse, M. R., Gerson, E. R., Veinot, J. P., \& Beller, C. J. (2013). Mechanical characterization of human aortas from pressurization testing and a paradigm shift for circumferential residual stress. Journal of the Mechanical Behavior of Biomedical Materials, 17, 44-55.

Langewouters, G. J., Wesseling, K. H., \& Goedhard, W. J. A. (1984). The static elastic properties of 45 human thoracic and 20 abdominal aortas in vitro and the parameters of a new model. Journal of Biomechanics, 17(6), 425-435.

Lanir, Y. (1983). Constitutive equations for fibrous connective tissues. Journal of Biomechanics, 16(1), 1-12.

Lee, J.Y., Jang, L.C., Sun, W.Y., Park, J.W., \& Choi, J.W. (2011). Relationship between tortuosity and atherosclerotic changes of the abdominal aorta. Korean Jounral of Vascular and Endovascular Surgery, 27(4):145-150.

Matsumoto, T., \& Hayashi, K. (1996). Stress and strain distribution in hypertensive and normotensive rat aorta considering residual strain. Journal of Biomechanical Engineering, 118(1), 62-71.

McEniery, C. M., Wilkinson, I. B., \& Avolio, A. P. (2007). Age, hypertension and arterial function. Clinical and Experimental Pharmacology and Physiology, 34(7), 665-671

Ogden, R. W., \& Saccomandi, G. (2007). Introducing mesoscopic information into constitutive equations for arterial walls. Biomechanics and Modeling in Mechanobiology, 6(5), 333-344.

O'Rourke, M. F., \& Hashimoto, J. (2007). Mechanical factors in arterial aging. A clinical perspective. Journal of the American College of Cardiology, 50(1), 1-13.

Persy, V., \& D'Haese, P. (2009). Vascular calcification and bone disease: The calcification paradox. Trends in Molecular Medicine, 15(9), 405-416.

Polzer, S., Gasser, T. C., Forsell, C., Druckmüllerova, H., Tichy, M., Staffa, R., Vlachovsky, R., \& Bursa, J. (2013). Automatic identification and validation of planar collagen organization in the aorta wall with application to abdominal aortic aneurysm. Microscopy and Microanalysis, 19(6), 1395-1404.

Pyle, A. L., \& Young, P. P. (2010). Atheromas feel the pressure: Biomechanical stress and atherosclerosis. American Journal of Pathology, 177(1), 4-9.

Rachev, A., \& Greenwald, S. E. (2003). Residual strains in conduit arteries. Journal of Biomechanics, 36(5), 661-670.

Roccabianca, S., Figueroa, C. A., Tellides, G., \& Humphrey, J. D. (2014). Quantification of regional differences in aortic stiffness in the aging human. Journal of the Mechanical Behavior of Biomedical Materials, 29, 618634.

Schriefl, A. J., Reinisch, A. J., Sankaran, S., Pierce, D. M., \& Holzapfel, G. A. (2012). Quantitative assessment of collagen fibre orientations from two-dimensional images of soft biological tissues. Journal of the Royal Society Interface, 9(76), 3081-3093.

Taber, L. A. (2004) Nonlinear theory of elasticity: Applications in biomechanics. World Scientific Publishing, Singapore.

Thubrikar, M. (2007). Vascular mechanics and pathology. Springer Science+Business Media, New York.

Treloar, L.R.G. (2005) The physics of rubber elasticity. Oxford University Press, Oxford, Third edition.

Valentín, A., Humphrey, J. D., \& Holzapfel, G. A. (2011). A multi-layered computational model of coupled elastin degradation, vasoactive dysfunction, and collagenous stiffening in aortic aging. Annals of Biomedical Engineering, 39(7), 2027-2045.

Wagenseil, J. E. (2011). A constrained mixture model for developing mouse aorta. Biomechanics and Modeling in Mechanobiology, 10(5), 671-687.

Weisbecker, H., Pierce, D. M., Regitnig, P., \& Holzapfel, G. A. (2012). Layer-specific damage experiments and modeling of human thoracic and abdominal aortas with non-atherosclerotic intimal thickening. Journal of the Mechanical Behavior of Biomedical Materials, 12, 93-106.

Wenn, C. M., \& Newman, D. L. (1990). Arterial tortuosity. Australasian Physical and Engineering Sciences in Medicine, 13(2), 67-70.

Wittek, A., Karatolios, K., Bihari, P., Schmitz-Rixen, T., Moosdorf, R., Vogt, S., \& Blase, C. (2013). In vivo determination of elastic properties of the human aorta based on 4D ultrasound data. Journal of the Mechanical Behavior of Biomedical Materials, 27, 167-183.

Wuyts, F. L., Vanhuyse, V. J., Langewouters, G. J., Decraemer, W. F., Raman, E. R., \& Buyle, S. (1995). Elastic properties of human aortas in relation to age and atherosclerosis: A structural model. Physics in Medicine and Biology, 40(10), 1577-1597.

Zulliger, M. A., \& Stergiopulos, N. (2007). Structural strain energy function applied to the ageing of the human aorta. Journal of Biomechanics, 40(14), 3061-3069. 